\title{
INLET AND OUTLET OPEN BOUNDARY CONDITIONS FOR INCOMPRESSIBLE NAVIER-STOKES EQUATIONS
}

\author{
C. Galusinski ${ }^{1}$, C. Mazoyer ${ }^{1,2}$, S. Meradji ${ }^{1}$, A. Molcard ${ }^{2}$, Y. Ourmières ${ }^{2}$ \\ ${ }^{1}$ IMATH, Université de Toulon, CS 60584, 83041 Toulon Cedex 9, France \\ 2 Université de Toulon, UMR7294, CNRS/INSU, IRD Mediterranean Institute of \\ Oceanography (MIO), UM 110, 83957 La Garde, France
}

\begin{abstract}
Flow simulation models often contain open boundaries in order to avoid computations on too large or infinite domains. The open boundary conditions partially drives the flow and the influence of the outer domain has to be taken into account on such boundaries. "Free" open boundary conditions are then essential to the model. The goal of this work is to introduce a new open boundary condition, adapted both for inlet and outlet, parametrized and driven by simple informations such as flow rate.
\end{abstract}

Keywords: Navier-Stokes equations, open boundary conditions.

\section{Introduction}

The present work deals with the choice of open boundary conditions for incompressible NavierStokes flows. Such a problematic is well understood for output flows even if the flow can re-enter intermittently into the domain in areas globally identified as output boundaries. The "Do nothing" boundary conditions $[1,2]$ lead to well-posed problem and work well on numerical experiments. They are nevertheless based on a reference velocity flow and a reference pressure and such data have to be well chosen. Such a method can be extended to open boundary conditions where the flow is permanently entering but limitations can be found in the choice of the reference velocity and pressure. This point is developed in the following part, leading to the proposition of another open boundary condition avoiding as much as possible the dependence to a reference flow. Concerning the reference pressure, it appears to be a strongly constraining information as pressure can vary in a spectacular way in turbulent flows and can not be intuited.

The goal of this work is to identify pertinent informations to define the open boundary conditions both for output and input boundaries. The key point is to let a free open boundary at inlet (where the flow is durably entering) by assuming that the dynamics is driven by forces inside the studied domain. Then a criterion on energy exchange, between the studied domain and the outside will be proposed. Another scalar parameter, the flow rate, will be another pertinent information linked to the energy exchange.

\subsection{Navier-Stokes equation and "do nothing" open boundary condi- tions.}

For the present study, the Navier-Stokes equations solving an incompressible viscous flow is used in an adimensional formulation,

$$
\begin{aligned}
R e\left(\frac{\partial \mathbf{u}}{\partial t}+\mathbf{u} \cdot \nabla \mathbf{u}\right)-\Delta \mathbf{u}+\nabla p=\mathbf{F} & \forall(t, \mathbf{x}) \in \mathbb{R}^{+} \times \Omega, \\
\nabla \cdot \mathbf{u}=0 & \forall(t, \mathbf{x}) \in \mathbb{R}^{+} \times \Omega,
\end{aligned}
$$

where $\mathbf{u}$ denotes the velocity, $p$ the pressure, $\mathbf{F}$ is a source term and $R e$ is the Reynolds number. The spatial domain $\Omega$ is bounded, surrounded by walls but also by open boundaries. Let us denote the boundary $\partial \Omega=\Gamma_{w} \cup \Gamma_{o}$ where $\Gamma_{w}$ corresponds to walls and $\Gamma_{o}$ to open boundaries.

The system is then closed with boundary conditions, including a no-slip boundary condition on the walls $\Gamma_{w}$ and an artificial boundary condition $(\mathrm{OBC})$ on $\Gamma_{o}$ which is the main matter of interest 
in the present work. The ways to close the system with an OBC leading to a well-posed problem are not so numerous. A natural approach is to impose a normal constraint to the boundaries but the inertia term (a nonlinear transport term) plays an important role when the flow enters into the domain and has then to be taken into account. Therefore, in [1, 2], the authors proposed an inhomogeneous Dirichlet boundary condition at the inlet and a nonlinear Robin boundary condition at the outlet. The boundary $\Gamma_{o}$ is then splitted in two parts,

$$
\Gamma_{o}=\Gamma_{\text {in }} \cup \Gamma_{\text {out }} .
$$

A reference flow and a reference pressure are then chosen, $\left(\mathbf{u}_{\mathbf{r}}, p_{r}\right)$ and the boundary condition writes

$$
\begin{aligned}
& \mathbf{u}=\mathbf{u}_{\mathbf{r}} \text { on } \Gamma_{i n} \\
& \partial_{\mathbf{n}} \mathbf{v}-q \mathbf{n}+\frac{R e}{2}(\mathbf{u} \cdot \mathbf{n})^{-} \mathbf{v}=0, \text { on } \Gamma_{\text {out }}, \\
& \text { with } \mathbf{u}=\mathbf{u}_{\mathbf{r}}+\mathbf{v}, \quad p=p_{r}+q,
\end{aligned}
$$

where $x^{-}=\max (0,-x)$. The problem $(1),(2),(3)$ and (4) studied in [1] is then well-posed. The choice of $\left(\mathbf{u}_{\mathbf{r}}, p_{r}\right)$ on $\Gamma_{\text {out }}$ is important for the simulations and works well when chosen from the solution at the previous time step.

In order to let free the flow at the inlet, the first idea is to use the OBC on $\Gamma_{\text {out }}$ for the boundary $\Gamma_{i n}$. On this boundary $\Gamma_{i n}$, where the flow has to be lastingly entering, the nonlinear Robin condition is durably applied to the reference flow up to a perturbation due to the reference pressure. For high Reynolds numbers, this boundary condition degenerates to an unknown Dirichlet boundary condition on $\Gamma_{i n}$. This inhomogeneous Dirichlet boundary condition is unknown since the pressure can be far from the reference pressure and the velocity far from the reference velocity $\mathbf{u}_{\mathbf{r}}$ concomitantly.

We then focus on OBCs that avoid the choice of a reference flow, and most importantly, a reference pressure. This is possible thanks to a splitting technique widely used to approach incompressible Navier-Stokes equations [5]. This is done for downstream OBCs, for example, in [3]. At one time step, a velocity prediction is computed thanks to the momentum equation, for a given pressure. Then, the predicted velocity is not divergence free and a projection onto the free divergence field allows the construction of a pressure for the next time step. For the prediction step, a free boundary condition is necessary only for the velocity. For the projection step defining the pressure, no reference pressure is necessary. In the absence of $\mathrm{OBC}$, this projection is unique and orthogonal, but with an $\mathrm{OBC}$, it is not the case and a wide choice of projection onto the free divergence field is possible. Our choice will be detailed in section 2. We first exhibit pertinent informations that will be the starting point to construct the correct projection.

\subsection{Criteria to control the flow}

As discussed in the previous section, when the OBC can be splitted between a region of durably ingoing flow $\Gamma_{\text {in }}$ and a region of globally outgoing flow $\Gamma_{\text {out }}$, the first goal is to avoid a Dirichlet boundary condition on $\Gamma_{i n}$. A flow rate can then be imposed instead. This information can not close the system, as it is simply a scalar information which can be pertinent and imposed as a property of the projected velocity on the free divergence field. This lets a lot of latitude to the projection.

Before detailing the projection, let us introduce another pertinent scalar parameter, the energy exchange between the studied domain and the outside. To define such an energy, let us remark that on a bounded domain $\Omega$ with $\partial \Omega=\Gamma_{w}$, the solution to (1) verifies

$$
\int_{\Omega} \nabla p \cdot \mathbf{u} d x=-\int_{\Omega} p \nabla \cdot \mathbf{u} d x+\int_{\Gamma_{w}} p \mathbf{u} \cdot \mathbf{n} d \gamma=0 .
$$

This shows that $\nabla p$ can be seen as a force that does not work globally on such a domain or an infinite domain. This means that, for a subdomain $\Theta \subset \Omega$, the quantity

$$
\int_{\Theta} \nabla p \cdot \mathbf{u} d x=-\int_{\Omega \backslash \Theta} \nabla p \cdot \mathbf{u} d x
$$


is exactly the energy exchange, due to the pressure, between the domain $\Theta$ and $\Omega \backslash \Theta$.

Returning to a domain $\Omega$ with walls and OBCs, the term

$$
e=\int_{\Omega} \nabla p \cdot \mathbf{u} d x=\int_{\Gamma_{o}} p \mathbf{u} \cdot \mathbf{n} d \gamma
$$

defines the energy exchange between the domain $\Omega$ and the outer domain. A pertinent scalar criterion can be to prohibit the energy exchange so that the flow dynamics is driven by the force inside $\Omega$ without additional energy due to the outside dynamics.

Because this condition seems difficult to impose, we choose to work with a criterion on the flow rate and to adapt this flow rate in order to modulate the energy $e$.

\section{Splitting method and OBC}

Let us recall, for simplicity, the projection method of order one, based on an order one time scheme with a time step $\delta t$. The upperscript $n$ refers to the $n^{t h}$ time iteration. The prediction step consists in finding the predicted velocity $\widetilde{\mathbf{u}}$ solving

$$
R e\left(\frac{\widetilde{\mathbf{u}}-\mathbf{u}^{n}}{\delta t}+\mathbf{u}^{\mathbf{n}} \cdot \nabla \mathbf{u}^{\mathbf{n}}\right)-\Delta \widetilde{\mathbf{u}}+\nabla p^{n}=\mathbf{F} \quad \forall \mathbf{x} \in \Omega,
$$

with additional boundary conditions on the velocity variable $\widetilde{\mathbf{u}}$, detailed herafter.

The correction step consists in finding the pressure $p^{n+1}$ and then the velocity $\mathbf{u}^{n+1}$ solution to

$$
\begin{aligned}
R e \frac{\mathbf{u}^{n+1}-\widetilde{\mathbf{u}}}{\delta t}-+\nabla\left(p^{n+1}-p^{n}\right)=0, & \forall \mathbf{x} \in \Omega, \\
\nabla \cdot \mathbf{u}^{n+1}=0 & \forall \mathbf{x} \in \Omega,
\end{aligned}
$$

with additional boundary conditions on $q=p^{n+1}-p^{n}$. It is worth noting that the $\mathbf{u}^{n+1}$ solution to $(7)$ is a projection of the predicted velocity $\widetilde{\mathbf{u}}$ onto the free divergence vector field, fixed by the choice of the boundary condition on $q$. By applying the operator $\nabla \cdot$ to $(7)$, the pressure difference $q$, solves the Poisson problem

$$
\frac{R e}{\delta t} \nabla \cdot \widetilde{\mathbf{u}}=\Delta q
$$

with the inhomogenous Neuman boundary condition we have chosen,

$$
\begin{array}{r}
\partial_{n} q=0, \text { on } \Gamma_{w}, \\
\partial_{n} q=\alpha \widetilde{\mathbf{u}} \cdot \mathbf{n}+\beta|\widetilde{\mathbf{u}} \cdot \mathbf{n}| \text { on } \Gamma_{o},
\end{array}
$$

where the constants $\alpha$ and $\beta$ are fixed with two scalar informations, the desired flow rate $d$ of $\mathbf{u}^{n+1}$ and the compatibility condition of the Poisson problem. This leads to

$$
\alpha=1+d \frac{\delta_{\text {out }}+\delta_{\text {in }}}{d_{\text {out }} \delta_{\text {in }}-d_{\text {in }} \delta_{\text {out }}}, \quad \beta=d \frac{d_{\text {out }}+d_{\text {in }}}{d_{\text {out }} \delta_{\text {in }}-d_{\text {in }} \delta_{\text {out }}},
$$

where

$$
d_{\text {in }}=\int_{\Gamma_{\text {in }}} \widetilde{\mathbf{u}} \cdot \mathbf{n} d \gamma, \quad d_{\text {out }}=\int_{\Gamma_{\text {out }}} \widetilde{\mathbf{u}} \cdot \mathbf{n} d \gamma, \quad \delta_{\text {in }}=\int_{\Gamma_{\text {in }}}|\widetilde{\mathbf{u}} \cdot \mathbf{n}| d \gamma, \quad \delta_{\text {out }}=\int_{\Gamma_{\text {out }}}|\widetilde{\mathbf{u}} \cdot \mathbf{n}| d \gamma .
$$

The choice (9) is motivated by modulations of $\widetilde{\mathbf{u}} \cdot \mathbf{n}$ on boundaries to construct $\mathbf{u}^{n+1}$ with the appropriate flow rate. The boundary conditions (9) (10) then closes the problem (7) with without introducing a reference pressure.

A variant of the system (7) (9), although less natural, is to gradually modulate the flow rate even if introducing a possible compressibility,

$$
\begin{array}{r}
\frac{R e}{\delta t}\left(\nabla \cdot \widetilde{\mathbf{u}}-(1-\theta) \int_{\Omega} \nabla \cdot \widetilde{\mathbf{u}} d x\right)=\Delta q, \\
\partial_{n} q=0, \text { on } \Gamma_{w}, \\
\partial_{n} q=\theta(\alpha \widetilde{\mathbf{u}} \cdot \mathbf{n}+\beta|\widetilde{\mathbf{u}} \cdot \mathbf{n}|) \text { on } \Gamma_{o},
\end{array}
$$


with $0 \leq \theta \leq 1$.

The next remaining task is then to close the boundary condition of (6). We choose a Robin like condition on open boundaries,

$$
\begin{array}{r}
\mathbf{u}=\mathbf{0} \text { on } \Gamma_{w} \\
\partial_{\mathbf{n}}\left(\widetilde{\mathbf{u}}-\mathbf{u}_{\mathbf{r}}\right)+k(\widetilde{\mathbf{u}} \cdot \mathbf{n})^{-}\left(\widetilde{\mathbf{u}}-\mathbf{u}_{\mathbf{r}}\right)=0, \text { on } \Gamma_{o},
\end{array}
$$

where the positive constant $k$ can be chosen to zero and a zero reference flow $\mathbf{u}_{\mathbf{r}}$ can be used. When $k$ is a large value, it enforces, where the flow enters the domain, the velocity to the reference flow $\mathbf{u}_{\mathbf{r}}$ which flow rate should be $d$ for compatibility.

The next section devoted to numerical experiments, allows discussions on the influence of $\theta$. In what follows, the parameter $k$ will be kept to zero with a trivial reference flow $\mathbf{u}_{\mathbf{r}}=0$. It is important to note that no reference pressure arises and only a reference flow rate drives the flow.

\section{Experiments}

In the following, the computations are done with the computational code introduced in [4], in a classical geometry for test case: a 2D channel with a circular obstacle or a 3D channel with a ball as obstacle. The obstacle diameter value is equal to half of the channel section length value. Namely, the obstacle is taken into account, on a staggered grid [6], with an order two approximation, even if the grid is non body fitted [4]. The time discrete model (6) (7) is closed with the OBCs (11) (13) parametrized with $\theta$ and $k$. The Reynolds number is chosen high $\left(\sim 10^{5}\right)$ for $2 \mathrm{D}$ simulations to increase physical instabilities in order to bring out the robustness of the OBCs, even if the grid size is not fine enough to produce relevant flows. The Reynolds number is chosen of order $10^{3}$ for 3D simulations.

The force $\mathbf{F}$ is chosen to a fixed value along the direction of the channel. At the inlet before the obstacle, the flow is expected laminar driven by such forces and we are concerned with flow rate which are compatible with such forces. We then choose the flow rate so that the energy exchange (5) is sufficiently small. We also will investigate some experiments with larger flow rates in order to evaluate the augmented energy exchange. The figure 1 is an example of an expected flow driven by the external force $\mathbf{F}$ with low energy exchanges. The reference flow rate for low energy exchange is 0.0275 for our geometry and force $\mathbf{F}$.

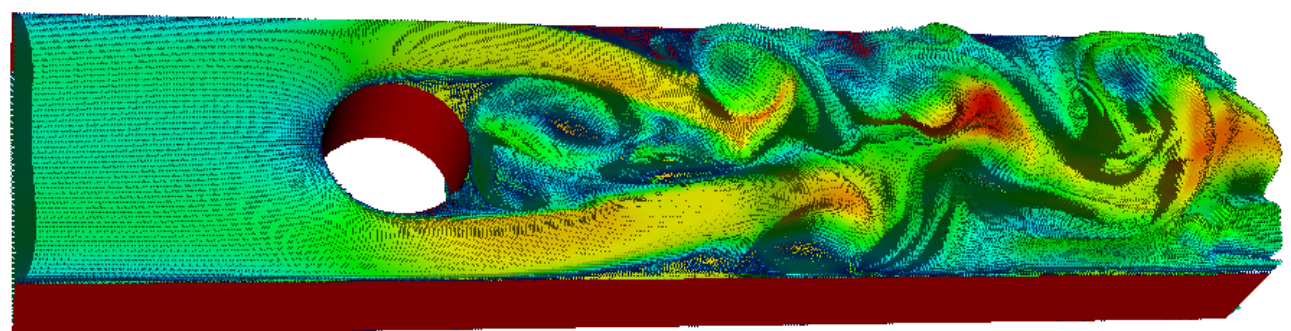

Figure 1: Example of expected flows.

We first investigate low value of $\theta$, namely $\theta=\frac{1}{400}$, with an imposed flow rate equal to 0.0275 . Even if $\theta$ is low, it is sufficient to lead the flow close to the desired flow rate and to respect the free divergence condition. The energy exchange oscillates around zero, leading to a low energy exchange, revealing a flow rate compatible with forces $\mathbf{F}$. The scale of the energy is proportional to the Reynolds number. It is also worth noting that the transitional regime occurs until time around 0.8 . 

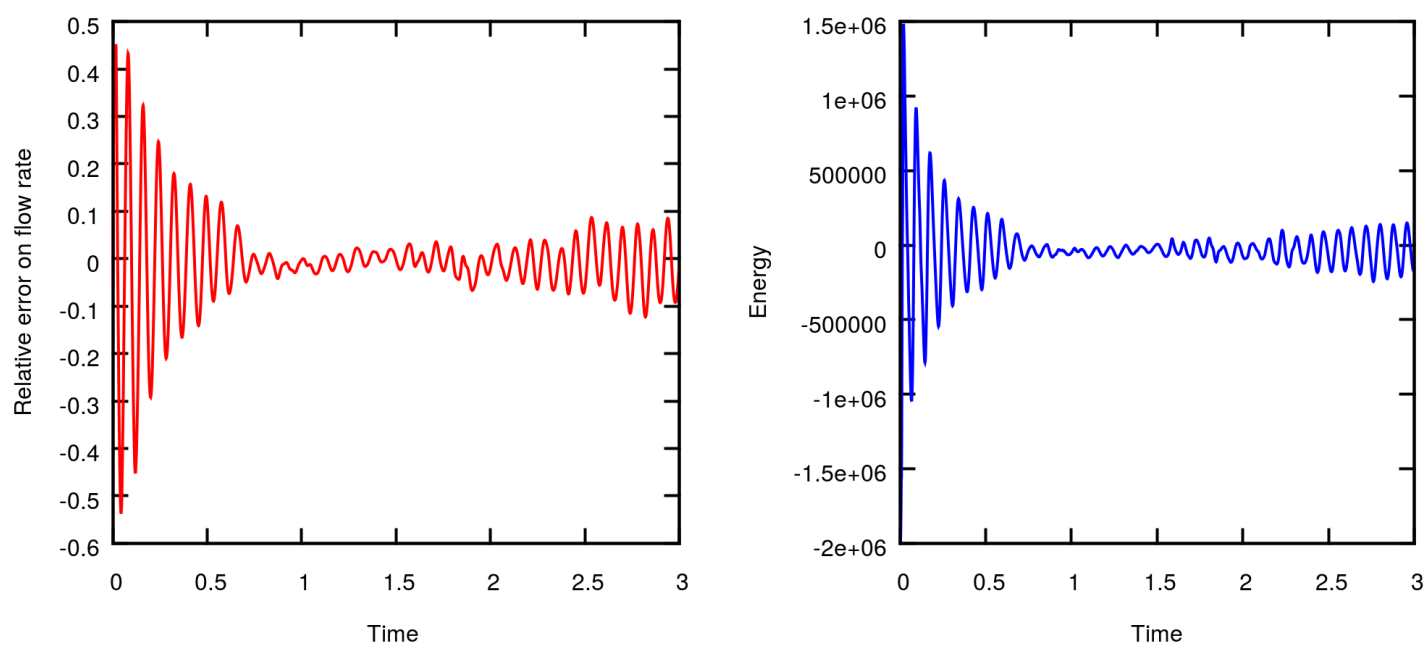

Figure 2: Flow rate relative error (left plot) and energy (right plot) with $\theta=\frac{1}{400}$

The influence of the expected flow rate value is now studied. Two simulations are analyzed: the first one with a reference flow rate of low energy exchange, and the second one with this flow rate multiplied by two. In the first time (before 0.5 ), flow rates and energy go through a transitional regime (figure 3): during this regime, the flow rate of low energy exchange has less important energy instabilities, that quickly stabilize. Then, after this transitional regime, both flow rates remain close to the expected flow rates (figure4). Regarding the energy, the figure 4 highlights the fact that a larger flow rate induces a more energetic flow during the whole simulation.
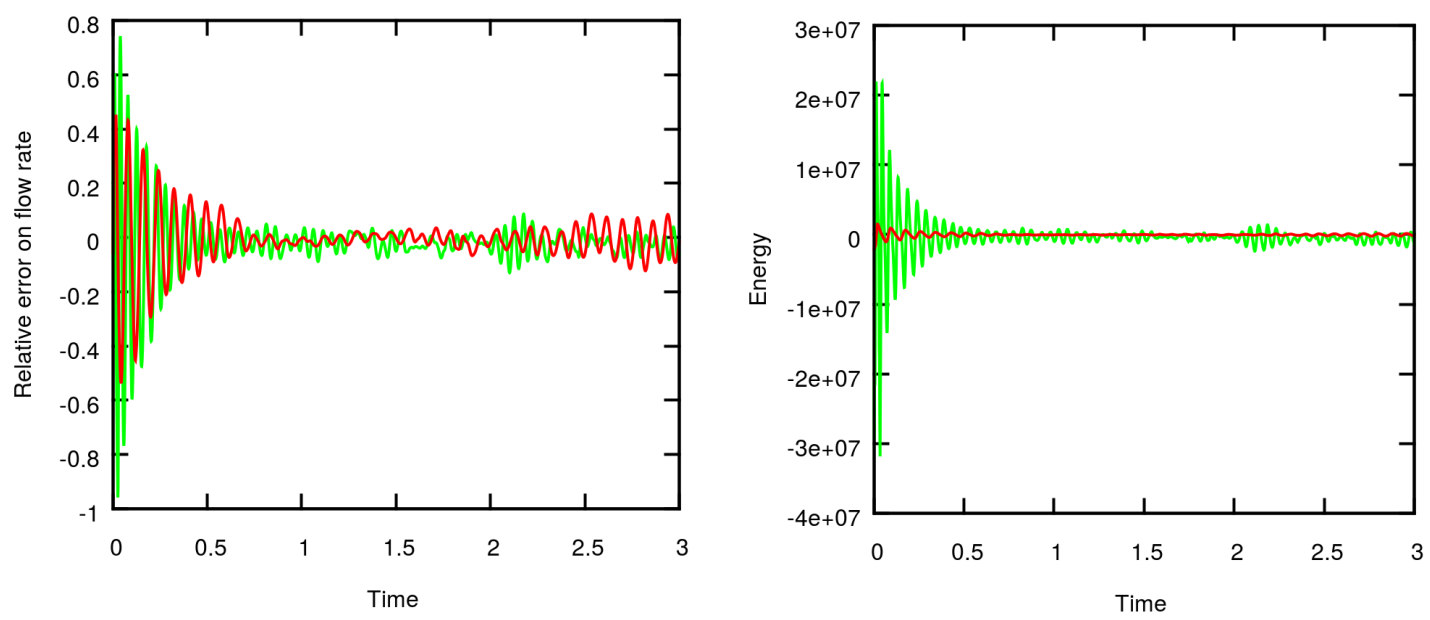

Figure 3: Reference flow rate influence ( 0.0275 (red color) and 0.055 (green color))on the observed flow rate (left plot) and the energy exchange (right plot). 

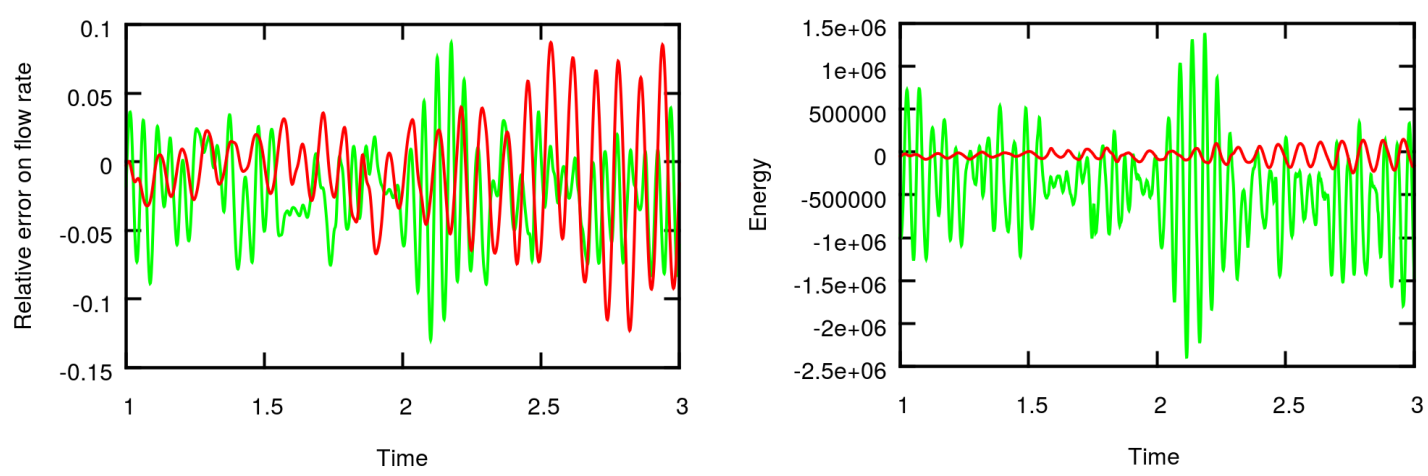

Figure 4: Reference flow rate influence (0.0275 (red color) and 0.055 (green color))on the observed flow rate and the energy exchange, skipping the transitional regime.

In the previous simulations, no reference flows are used and $k=0$ in (13). When taking $k$ large and a reference flow at the inlet, similar flows to those associated to the Figures 1 and 2 are obtained and values of $\theta$ are not sensitive.

In the following 3D simulations, larger values of $\theta$ are used, leading to very low variations on the flow rate, as shown in Figure 5. The value $\theta=1$ should lead to an exact flow rate but, as the divergence of the velocities is not exactly zero in simulations, very low variations on the flow rate occur. In this case, the energy exchange is negative and small compared to the Reynolds number value. This means that the reference flow rate is slightly greater than a zero exchange energy flow rate. As depicted in Figure 5, we can observe that the energy exchange is very weakly influenced by $\theta$ for $\frac{1}{10} \leq \theta \leq 1$, suggesting that flow rate variations are negligible.
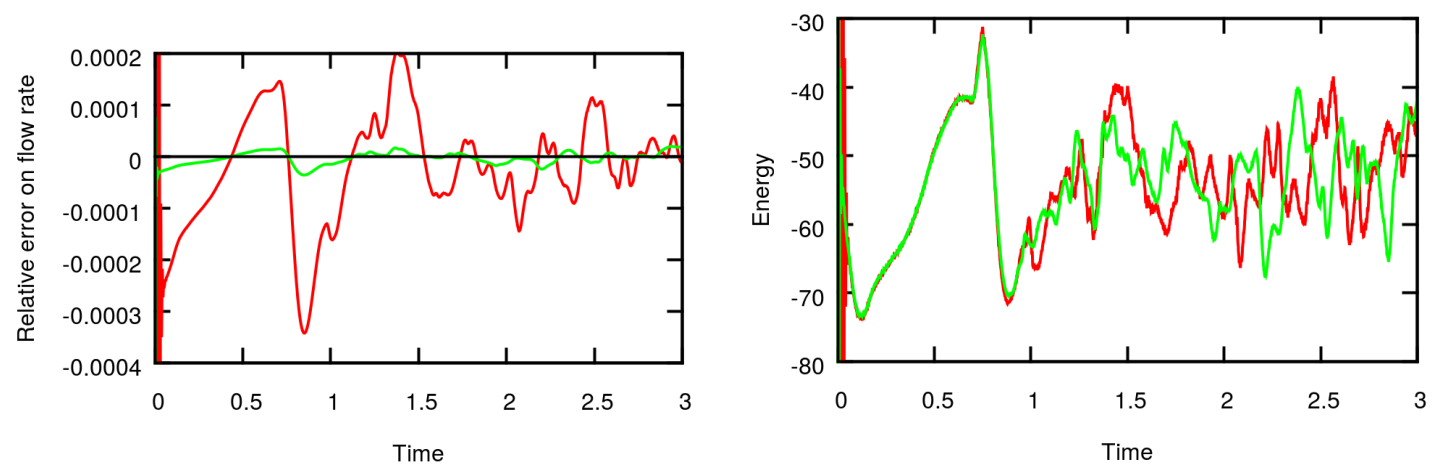

Figure 5: Influence of $\theta, \theta=1$ (green plots) and $\theta=\frac{1}{10}$ (red plots).

The Figure 6 shows the relative error on the flow rate of the Figure 5 multiplied by $\theta$. The curves are then very closed suggesting the linear behavior of the relative error on the flow rate with respect to $\theta$. Similar plots are added for a truncated channel. 

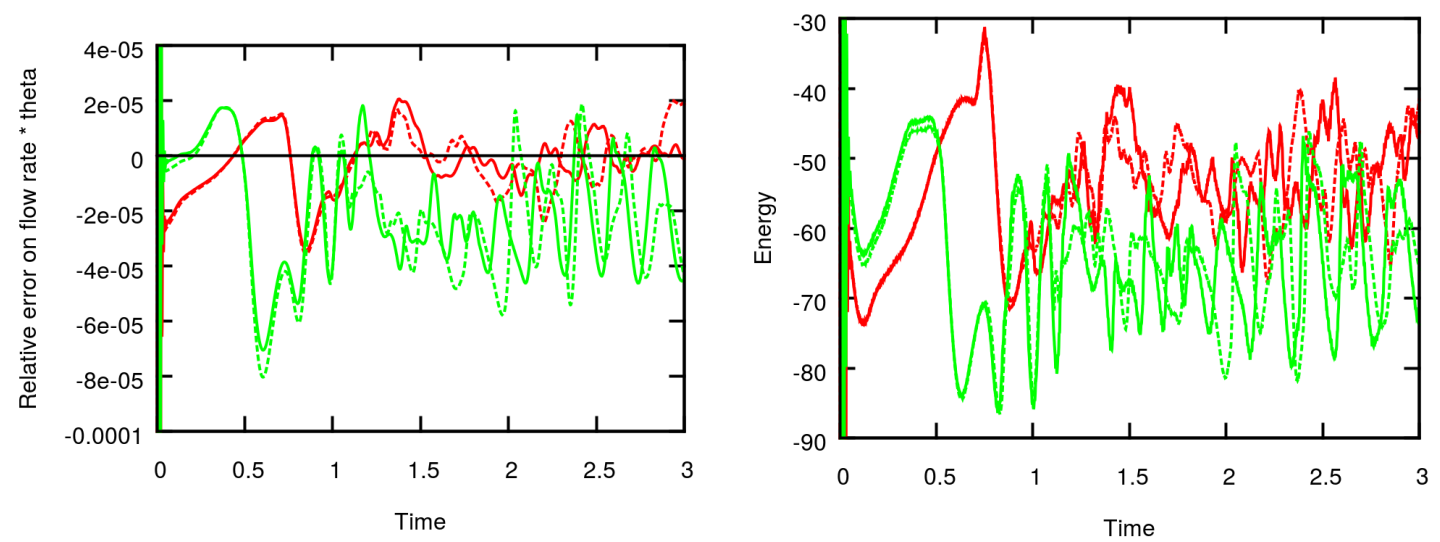

Figure 6: Flow rate relative error multiplied by $\theta$ on the left figure, energy exchange on the right figure. Plots related to a truncated channel are in green. Plots related to a standard channel are in red. Dashed lines represent $\theta=1$, and full lines represent $\theta=\frac{1}{10}$.

For $\theta=\frac{1}{10}$ or $\theta=1$, time $t=1$, the velocity solution of the $3 \mathrm{D}$ channel is introduced in Figures 7 and 8 , for the same flow rate. Figure 7 shows the velocity solution for a long channel and Figure 8 shows it for a shorter channel. Few differences can be observed on velocities because of the large time value chosen and the sensitivity of the flow.
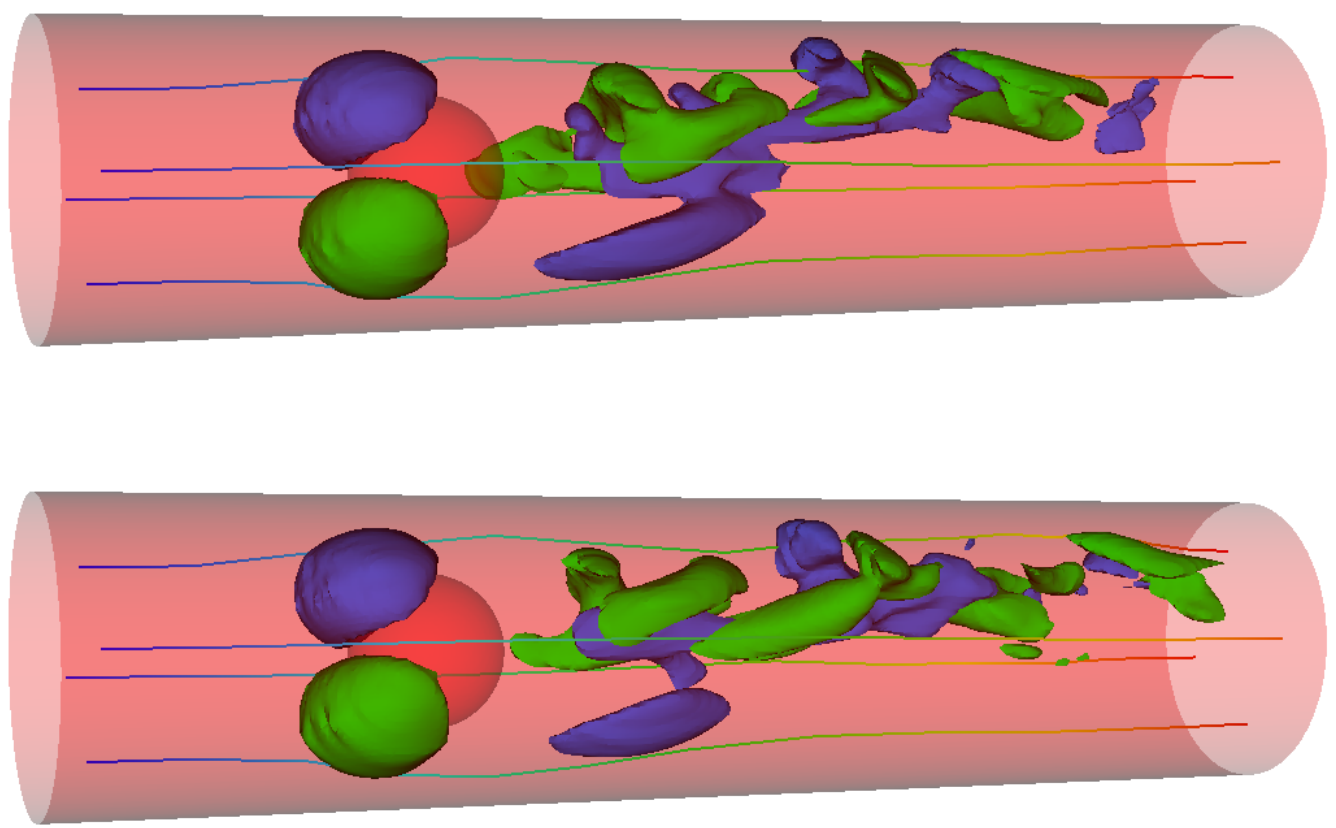

Figure 7: Streamlines and two isovalues of a spanwise component of the velocity: $\theta=\frac{1}{10}$ (top), $\theta=1$ (bottom). 

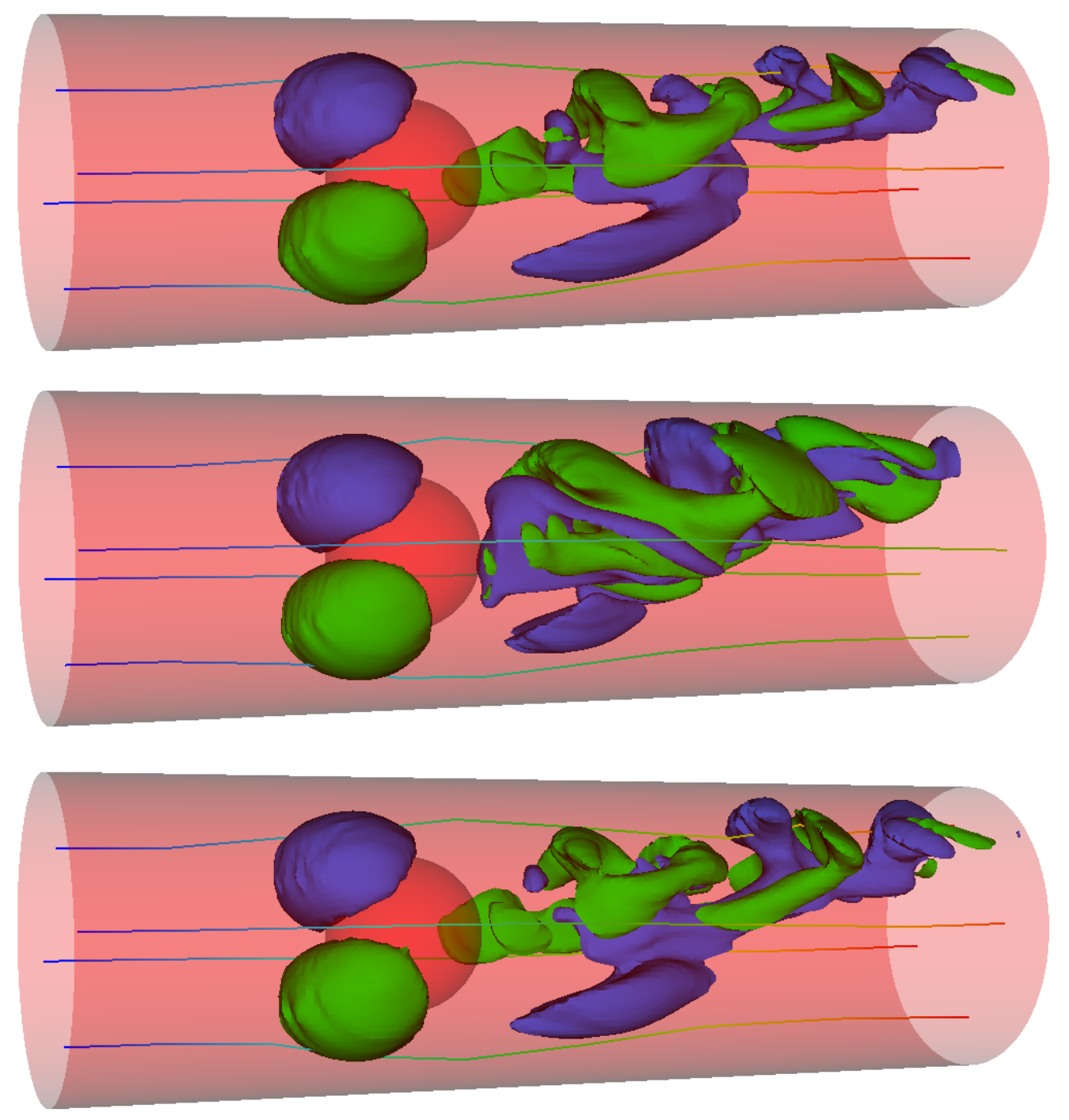

Figure 8: Streamlines and two isovalues of a spanwise component of the velocity: $\theta=\frac{1}{10}$ (top and middle), $\theta=1$ (bottom). Grid size is finer on the middle figure (90 nodes in the cross section of the channel instead of 60 ).

\section{Conclusions}

The new boundary condition introduced here allows to construct a flow driven by forces inside the geometry and by a given flow rate taking into account interactions with the outer domain. A notion of energy exchange has been introduced. The flow rate can be tuned to control the energy exchange between the domain and the outer domain. It has been evidenced that the exchange energy is strongly linked to the flow rate. If the model allows to impose a flow of reference at inlet, we prefer to eliminate the influence of a flow of reference by choosing $k=0$ in the boundary condition (13). The flow is then only driven by a scalar, the flow rate of reference. Furthermore, the model depends on an artificial parameter $\theta$ in (11) which allows to obtain precisely the desired flow rate for $\theta$ close to 1 or to oscillate around the desired flow rate for $\theta$ close to 0 .

In the continuity of this work, in a future development, another OBC could be proposed in order to ensure a free (or given) energy exchange in a direct way (without given flow rates) and should be compared to this one. Finally, our aim is to implement such OBCs in the context of ocean modelling for the simulation of reduced sea areas when the flow dynamics is potentially driven by local forces such as the wind. In such a case, the region considered as $\Gamma_{\text {in }}$ and $\Gamma_{\text {out }}$ are 
not as simple as the ones in a channel, but $\Gamma_{\text {in }}$ can be identified as the upstream frontier and $\Gamma_{\text {out }}$ as the downstream frontier, possibly changing or swapping with time.

Acknowledgments: This work was granted access to the HPC resources of Aix-Marseille Universit financed by the project Equip@Meso (ANR-10-EQPX-29-01) of the program Investissements d'Avenir supervised by the Agence Nationale de la Recherche.

\section{References}

[1] Bruneau, C-H and Fabrie, P: New efficient boundary conditions for incompressible NavierStokes equations: a well-posedness result, RAIRO-MMAN, vol. 30, (1996), N. 7, pp. 815-840.

[2] Bruneau, CH and Fabrie, P: Effective downstream boundary conditions for incompressible Navier-Stokes equations, International Journal for Numerical Methods in Fluids, vol. 19 (1994) n. 8, pp. $693-705$.

[3] Dong, S. and Shen, J.: A pressure correction scheme for generalized form of energy-stable open boundary conditions for incompressible flows. J. Comput. Phys., Journal of Computational Physics, vol. 291, (2015), pp. 254-278.

[4] Galusinski, Cedric: Problematic of Incompressible Bifluid Navier-Stokes Equations Approached on Non Body Fitted Meshes, Topical Problems of Fluid Mechanics (2016), pp. 51-58.

[5] J. L. Guermond and Jie Shen: An overview of projection methods for incompressible flows. Comput. Methods Appl. Mech. Engrg, vol. 41 (2006), pp. 112-134.

[6] Francis H. Harlow and J. Eddie Welch: Numerical calculation of time-dependent viscous incompressible flow of fluid with free surface. Physics of Fluids, vol. 8(12) (1965), pp. :21822189. 